\title{
Stratosphere-Troposphere Coupling in the Southern Hemisphere
}

\author{
DAVID W. J. THOMPSON \\ Department of Atmospheric Science, Colorado State University, Fort Collins, Colorado \\ MARK P. BALDWIN \\ Northwest Research Associates, Bellevue, Washington \\ SUSAN SOLOMON \\ National Oceanic and Atmospheric Administration/Aeronomy Laboratory, Boulder, Colorado
}

(Manuscript received 22 May 2003, in final form 27 January 2004)

\begin{abstract}
This study examines the temporal evolution of the tropospheric circulation following large-amplitude variations in the strength of the Southern Hemisphere ( $\mathrm{SH}$ ) stratospheric polar vortex in data from 1979 to 2001 and following the SH sudden stratospheric warming of 2002. In both cases, anomalies in the strength of the SH stratospheric polar vortex precede similarly signed anomalies in the tropospheric circulation that persist for more than 2 months. The SH tropospheric circulation anomalies reflect a bias in the polarity of the SH annular mode (SAM), a large-scale pattern of climate variability characterized by fluctuations in the strength of the SH circumpolar flow. Consistent with the climate impacts of the SAM, variations in the stratospheric polar vortex are also followed by coherent changes in surface temperatures throughout much of Antarctica. The results add to a growing body of evidence that suggests that stratospheric variability plays an important role in driving climate variability at Earth's surface on a range of time scales.
\end{abstract}

\section{Introduction}

The Southern Hemisphere ( $\mathrm{SH}$ ) sudden stratospheric warming (SSW) of 2002 was unique in more than four decades of observations not only in terms of its timing during the calendar year, but also in terms of its amplitude. Other papers in this volume deal extensively with the state of the tropospheric circulation during the period leading up to the SSW of 2002 and of the SH stratospheric circulation throughout the life cycle of the event (e.g., Charlton et al. 2005; Newman and Nash 2005; Orsolini et al. 2005). In this contribution, we focus on the relationship between the breakdown of the SH stratospheric flow and the state of the $\mathrm{SH}$ troposphere during the period immediately following the event.

The study is motivated by the recent finding that in the Northern Hemisphere ( $\mathrm{NH})$ winter, large-amplitude weakenings and strengthenings of the stratospheric polar vortex are typically followed by similarly signed anomalies in the tropospheric circulation that

Corresponding author address: David W. J. Thompson, Department of Atmospheric Science, Colorado State University, Fort Collins, CO 80523-1371.

E-mail: davet@atmos.colostate.edu persist for up to 2 months (Baldwin and Dunkerton 2001; Baldwin et al. 2003). The life cycle of these NH coupled vortex events is characterized as follows: 1) anomalous upward-propagating wave activity from the troposphere induces large anomalies in the $\mathrm{NH}$ stratospheric zonal flow (e.g., Zhou et al. 2002); 2) the anomalies in the NH stratospheric zonal flow descend over the course of $\sim 1-2$ weeks from the middle to the lower stratosphere, where they persist for up to $\sim 60$ days (Baldwin and Dunkerton 1999, 2001; Baldwin et al. 2003); 3) the growth of wind anomalies in the lowerstratospheric zonal flow coincides with the growth of anomalies in the eddy momentum flux in the upper troposphere (Limpasuvan et al. 2004); and 4) the anomalous eddy momentum flux in the upper troposphere induces anomalies in the tropospheric zonal flow that persist for as long as the overlying lowerstratospheric anomalies (Limpasuvan et al. 2004). Throughout the life cycle of these coupled vortex events, the anomalies in the stratospheric and tropospheric circulations strongly resemble the leading mode of variability in the NH circulation, the so-called Northern Hemisphere annular mode (Thompson and Wallace 2000; also referred to as the North Atlantic Oscillation and the Arctic Oscillation). 
The aforementioned papers focus on the state of the tropospheric circulation following large-amplitude stratospheric events in the Northern Hemisphere. In this study, we examine the state of the tropospheric circulation following analogous events in the Southern Hemisphere. The paper is divided into two parts. In the first part, we examine the mean evolution of the $\mathrm{SH}$ stratospheric and tropospheric circulations during the largest stratospheric events that occurred between 1979 and 2001. In the second part, we compare results based on events in 1979-2001 to those derived from the SH sudden stratospheric warming of September 2002.

\section{Analysis}

The principal data used in this study are daily average values of the National Centers for Environmental Prediction-National Center for Atmospheric Research reanalysis from 1 January 1979 to 31 December 2002 obtained from the National Oceanic and Atmospheric Administration Climate Diagnostics Center (Kalnay et al. 1996). The analysis is restricted to the period following 1979, which corresponds to the year when satellite data were included in the assimilation (Kalnay et al. 1996). We also use monthly mean temperatures from the Climate Research Unit at the University of East Anglia (Jones 1994).

The coupling between variations in the zonal flow of the SH stratosphere and troposphere is dominated by variations in the Southern Hemisphere annular mode (SAM). The structure of the SAM in the troposphere is discussed, for example, in Shiotani (1990), Kidson (1988), Karoly (1990), Hartmann and Lo (1998), Gong and Wang (1999), and Thompson and Wallace (2000). The coupling between month-to-month fluctuations in the SAM and the circulation of the $\mathrm{SH}$ stratosphere is discussed in Thompson and Wallace (2000). The SAM is defined separately at 17 pressure levels, $1000-10 \mathrm{hPa}$, as the leading empirical orthogonal function (EOF) of monthly mean geopotential height anomalies at $20^{\circ}-$ $90^{\circ} \mathrm{S}$ during September-December. The SeptemberDecember season is chosen because the SAM exhibits maximum variance in the stratosphere during the spring season (Thompson and Wallace 2000). At tropospheric levels, where the SAM exhibits considerable variance year-round, the resulting EOF weighting pattern is virtually identical to the leading EOF derived from data for all months of the year. Daily time series of the SAM are generated by projecting daily fields of geopotential height anomalies for each calendar day, 1979-2002, onto the respective EOF weighting patterns. The resulting time series are standardized so that the mean and variance of the time series at each level are 0 and 1, respectively, for the period 1979-2001. By convention, positive values of the SAM index are defined as anomalously westerly zonal flow along $60^{\circ} \mathrm{S}$ and anomalously low geopotential heights over the polar cap, and vice versa.

\section{Composite life cycle: 1979-2001}

Figure 1 shows the variance of the SAM index time series as a function of level and time of year (shading) superposed on the climatological strength of the zonal flow along $60^{\circ} \mathrm{S}$ (contours). In the troposphere, the variance of the SAM peaks during the winter season of May-October. The wintertime peak in the tropospheric variance of the SAM is consistent with the observations that 1) variations in the SAM are associated with interactions between waves and the mid-high-latitude zonal flow (Kidson 1988; Karoly 1990; Hartmann and Lo 1998; Thompson and Wallace 2000; Limpasuvan and Hartmann 2000; Lorenz and Hartmann 2001) and 2) tropospheric baroclinic activity is most vigorous during the winter season.

In the stratosphere, the variance in the SAM peaks during the spring season, with regions of maximum variance descending from the middle to lower stratosphere from September to December. The springtime peak in the stratospheric variance of the SAM reflects year-to-year variations in the timing of the breakdown of the SH stratospheric vortex, as evidenced by the fact that the stratospheric variance maxima closely track the rapid decrease in the strength of the zonal flow during the SH spring season. The apparent downward propagation of the seasonal breakdown of the SH stratospheric polar vortex is consistent with findings reported in numerous studies (e.g., Hartmann 1976; Mechoso 1988; Kuroda and Kodera 1998). As noted in Thompson and Wallace (2000), the timing of the stratospheric variance maxima in the SAM is consistent with the theoretical criteria for vertically propagating waves outlined in Charney and Drazin (1961), in which waves propagate vertically when the background zonal flow is westerly but less than a threshold value. As such, there is reduced wave activity in the $\mathrm{SH}$ stratosphere during midwinter when the stratospheric circumpolar flow is very strong but considerable wave activity in the $\mathrm{SH}$ stratosphere during the spring season as the vortex be-

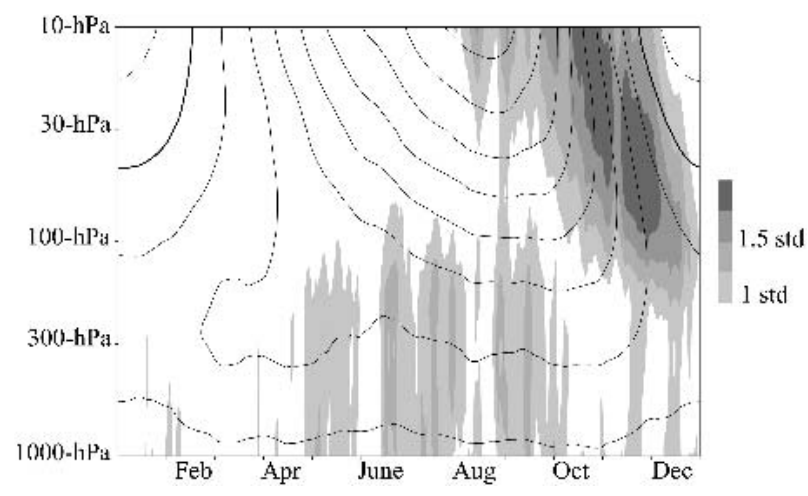

FIG. 1. Variance of the SAM index (shading) and the strength of the zonal-mean flow along $60^{\circ} \mathrm{S}$ (contours) as a function of calendar day and vertical level. Contours are drawn at $10 \mathrm{~m} \mathrm{~s}^{-1}$. Negative contours are dashed. The zero contour is thickened. 
gins its seasonal decline. The absence of a secondary variance maximum during the fall season is consistent with the broad meridional scale of the stratospheric polar vortex at this time, conditions that render the vortex less susceptible to vertically propagating waves (e.g., McIntyre 1982).

The results in Fig. 1 illustrate that large-amplitude variations in the $\mathrm{SH}$ stratospheric flow occur primarily during the late winter/spring season. We examine the temporal evolution of such variations by compositing the SAM index during strengthenings and weakenings of the stratospheric polar vortex, referred to hereafter as strong and weak stratospheric events. Strong and weak stratospheric events are defined as the seven largest positive and negative anomalies in the SAM index at $10 \mathrm{hPa}$ between 1979 and $2001(10 \mathrm{hPa}$ is the highest level available in the reanalysis). Since the length of the active season for wave-mean-flow interactions in the SH stratosphere is comparable to the time scale of variability there (Baldwin et al. 2003), we consider only the largest event of each sign during each calendar year. Following Baldwin and Dunkerton (2001), we composite about the onset date of the stratospheric events, in which the onset dates are defined here as the day when anomalies in the SAM index cross the two-standarddeviation threshold for the final time prior to the peak of the event ( -2 for weakenings, +2 for strengthenings).

The onset dates, peak dates, and peak amplitude of the events used in the composite analysis are listed in Table 1 . The analysis permits events during any time of year, but, consistent with Fig. 1, all of the events occur during the SH late winter/spring season. Note that the events used in the analysis correspond closely to terciles of the data during the spring season. In contrast to similar results calculated for the NH (e.g., Baldwin and Dunkerton 2001), the results in this study are derived from a relatively small sample. This is because the most comprehensive observations of the SH stratosphere are restricted to the period following 1979, and because the SH stratosphere typically exhibits only one event during a single active season. To maximize the sample size used in the analyses, we examine the difference between the means for the weak and strong composites, rather than the separate composites themselves. The results are not sensitive to the details of the analysis: similar results were found for composites based on different thresholds, on the peak (rather than onset) dates and on detrended data. The statistical significance of the results is estimated using the $t$ statistic for the difference in means between two samples (e.g., von Storch and Zwiers 1999, their section 6.6). Since results for the $\mathrm{NH}$ provide an a priori expectation of the sign of the results, confidence levels are based on a one-tailed test of the difference in sample means.

Figure 2 shows values of the SAM index composited about the onset dates listed in Table 1 for levels 1000$10 \mathrm{hPa}$ and lags -90 to +90 days, where day 0 corresponds to the onset date. By construction, the largest anomalies are found at $10 \mathrm{hPa}$, where the $\mathrm{SAM}$ is more than four standard deviations lower during the first week following the onset of weak events than it is during the first week following the onset of strong events. The anomalies peak shortly after day 0 in the middle stratosphere and subsequently descend with positive lag to the lower stratosphere. The descent of anomalies within the SH stratosphere is consistent with findings reported in Kuroda and Kodera (1998) and Graversen and Christiansen (2003). In the lower stratosphere, anomalies in excess of one standard deviation are observed for close to 3 months following the onset of the event, somewhat longer than is observed in association with analogous events in the NH (Baldwin and Dunkerton 2001).

The results in Fig. 2 reveal that large-amplitude variations in the SH stratospheric polar vortex are followed by persistent anomalies not only in the stratospheric circulation, but in the tropospheric circulation as well. On average, the $\sim 3$-month period following the onset of the composite stratospheric event is associated with tropospheric anomalies that have the same sign as the overlying stratospheric anomalies. The shift in the tropospheric circulation is also apparent in the probability distribution functions (PDFs) calculated for daily values of the SAM index at $500 \mathrm{hPa}$ during the 10-90-day period following the seven weakenings (Fig. 3, left; light line) and strengthenings (Fig. 3, left; dark line) of the SH stratospheric polar vortex listed in Table 1 . The period of $10-90$ days was chosen since 1) 10 days correspond roughly to the limit of deterministic

TABLE 1. Weak and strong stratospheric events used in this study. Amplitude is in std dev of the SAM index at $10 \mathrm{hPa}$.

\begin{tabular}{|c|c|c|c|c|c|c|c|}
\hline \multicolumn{4}{|c|}{ Weak events } & \multicolumn{4}{|c|}{ Strong events } \\
\hline Year & Onset date & Peak date & Peak amplitude & Year & Onset date & Peak date & Peak amplitude \\
\hline 1988 & $1 \mathrm{Aug}$ & 31 Aug & -5.9 & 1987 & $13 \mathrm{Nov}$ & $16 \mathrm{Nov}$ & 3.9 \\
\hline 1979 & $1 \mathrm{Oct}$ & 17 Oct & -4.8 & 1985 & 10 Oct & 13 Oct & 3.2 \\
\hline 1996 & $4 \mathrm{Aug}$ & $12 \mathrm{Aug}$ & -3.8 & 1996 & 31 Oct & $5 \mathrm{Nov}$ & 2.8 \\
\hline 2000 & 17 Oct & $3 \mathrm{Nov}$ & -3.4 & 1998 & 19 Nov & $26 \mathrm{Nov}$ & 2.5 \\
\hline 1991 & $22 \mathrm{Oct}$ & $27 \mathrm{Oct}$ & -3.2 & 1997 & $21 \mathrm{Oct}$ & $25 \mathrm{Oct}$ & 2.5 \\
\hline 1992 & $28 \mathrm{Sep}$ & $1 \mathrm{Oct}$ & -3.0 & 1999 & $22 \mathrm{Oct}$ & 27 Oct & 2.5 \\
\hline 1995 & 11 Sep & $14 \mathrm{Sep}$ & -2.9 & 1995 & $4 \mathrm{Sep}$ & 4 Oct & 2.4 \\
\hline 2002 & 30 Aug & 27 Sep & -9.8 & & & & \\
\hline
\end{tabular}




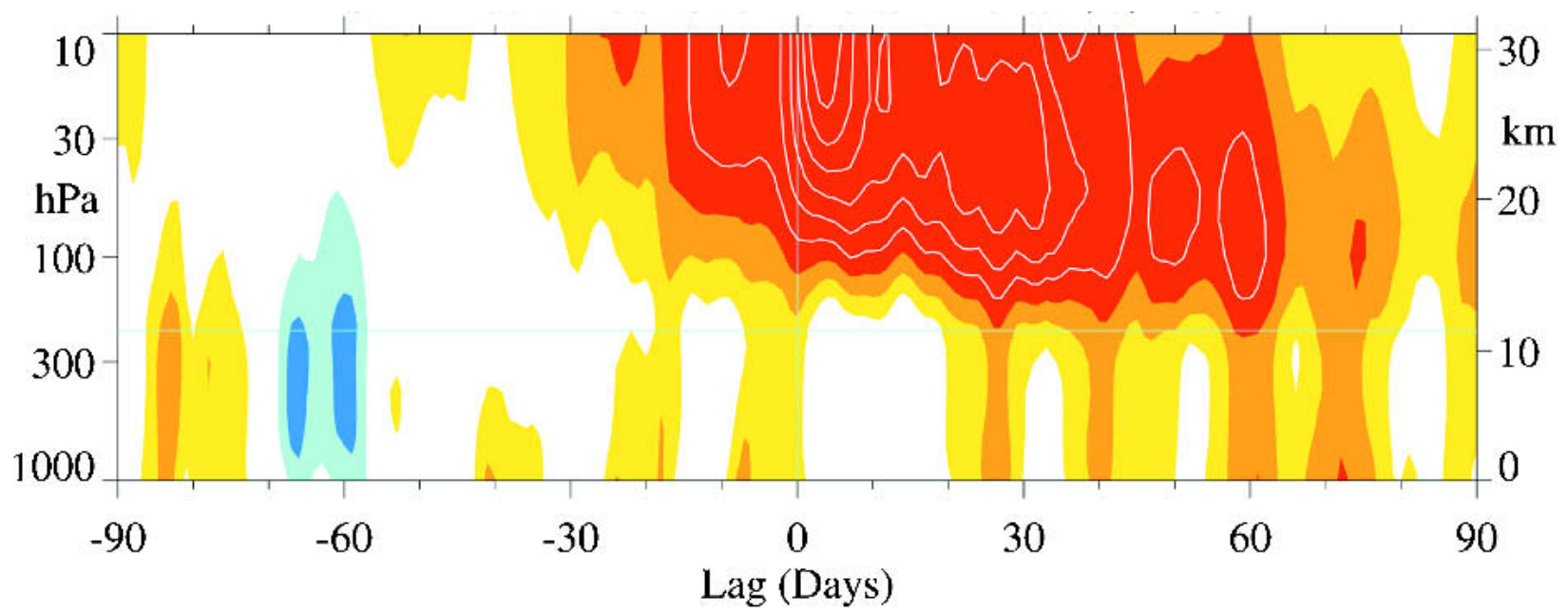

FIG. 2. Composite difference of the SAM index between the weak and strong stratospheric events listed in Table 1 . Day 0 corresponds to the onset of the stratospheric event at $10 \mathrm{hPa}$. Contour and shading interval is at $0.5 \mathrm{std}$ dev. Shading is drawn for values exceeding \pm 0.5 std dev. Red shading denotes positive values in the SAM index (weaker-than-normal zonal flow along $60^{\circ} \mathrm{S}$ ).

weather forecasts and 2) the lower-stratospheric and tropospheric features in Fig. 2 persist for $\sim 3$ months. As is evident in the figure, the 10-90-day period following weakenings of the vortex is associated with daily values of the tropospheric SAM index values that are on average $\sim 0.7$ standard deviations lower than those found during the 10-90-day period following strengthenings of the vortex, a difference that exceeds the $95 \%$ confidence level. The significance of this result is not sensitive to the specific time interval chosen.

The shifts in the SAM evident in Fig. 3 are associated with substantial anomalies in the $500-\mathrm{hPa}$ geopotential height field. Figure 4 (middle) shows the difference in the $500-\mathrm{hPa}$ height field between the 10-90-day periods following weak and strong events at $10 \mathrm{hPa}$, respectively (i.e., the figure is based on the same samples used in Fig. 3). Figure 4 (left) shows the structure of the $\mathrm{SAM}$ in the month-to-month variability, defined here by regressing the $500-\mathrm{hPa}$ height field onto monthly mean values of the SAM index at $500 \mathrm{hPa}$. The results in Fig. 4 (left) are inverted, such that positive values correspond to conditions associated with the low index polarity of the SAM, and vice versa. As is evident in Fig. 4 (middle), major weakenings in the $\mathrm{SH}$ stratospheric polar vortex are followed by statistically significant rises in tropospheric geopotential heights throughout the polar cap and falls in geopotential heights at midlatitudes that bear a strong resemblance to the structure of the SAM in the month-to-month variability. The spatial correlation between the structures in Fig. 4 (left) and Fig. 4 (middle) is $r=0.85$.

What are the climate impacts of the tropospheric anomalies evident in Figs. 2-4? At tropospheric levels, the SAM is coupled with variations in surface temperature throughout Antarctica (Thompson and Wallace 2000; Thompson and Solomon 2002). Its high index po-
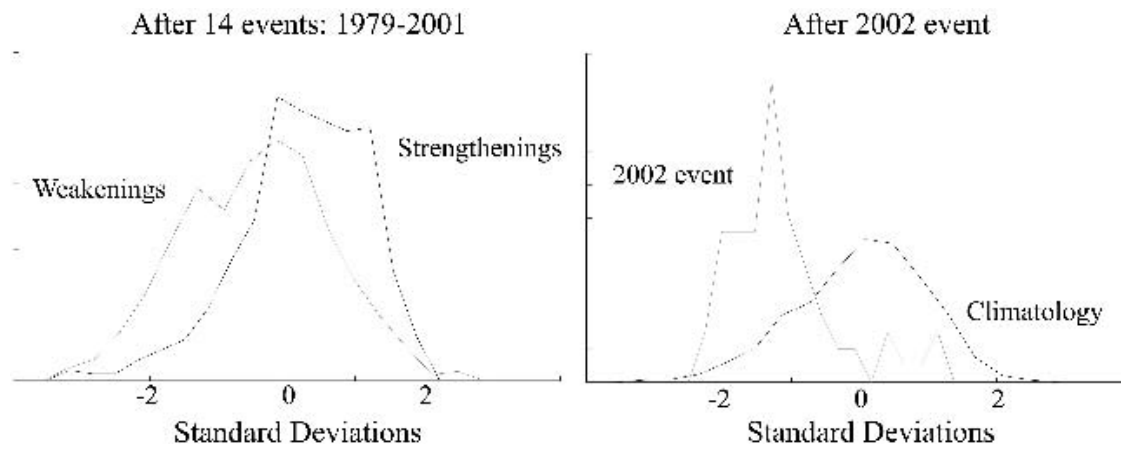

FIG. 3. (left) Histograms of the SAM index at 500-hPa during the 10-90-day period following the onset of weak (gray) and strong (black) stratospheric events. The events are listed in Table 1. (right) As in the left, but for the 10-90-day period following the onset of the $2002 \mathrm{SH}$ SSW (gray) and the Oct-Nov climatology (black). 

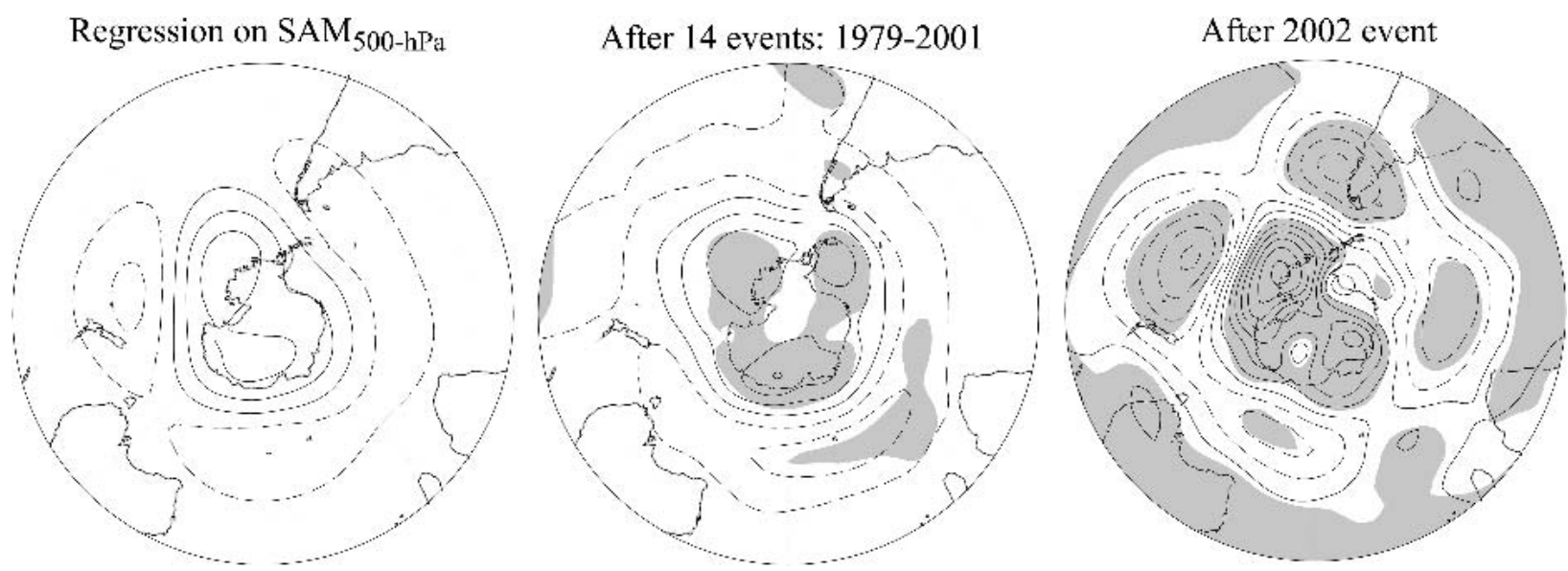

FIG. 4. (left) Monthly mean values of 500-hPa height regressed on standardized values of the SAM index at $500 \mathrm{hPa}$. The regression coefficients are inverted such that negative values of the SAM index correspond to higher-than-normal heights over the Pole, and vice versa. (middle) Composite difference between 500-hPa heights during the 10-90-day period following the onset of the weak and strong stratospheric events listed in Table 1. (right) 500-hPa geopotential height anomalies during the 10-90-day period following the onset of the 2002 SH SSW. Contours are drawn at $20 \mathrm{~m}(-10,10,30, \ldots)$. Shading denotes regions where the results exceed the (middle) $95 \%$ confidence level and (right) 1 std dev about the climatological mean.

larity is associated with lower-than-normal temperatures over eastern Antarctica and the Antarctic Plateau and by relatively weak warm anomalies over the Antarctic Peninsula; its low index is marked by anomalies in the opposite sense. Hence, it follows that weakenings of the vortex should be followed by warmer-thannormal temperatures over much of Antarctica with the exception of the Peninsula region, and vice versa. Figure 5 demonstrates that this is, in fact, the case.

Figure 5 (left) shows surface temperature anomalies regressed onto the monthly mean time series of the SAM. As in Fig. 4 (left), the results are inverted such that positive values correspond to conditions associated with the low index polarity of the SAM, and vice versa.
Figure 5 (middle) shows surface temperature anomalies over Antarctica averaged over the 3-month period following the peak of the event in the stratosphere. Since the surface temperature observations used in this study are available in monthly mean form, the 3-month period starts on the first day of the first month following the onset date of the stratospheric event; for example, if the onset of the stratospheric event occurred during September, the surface temperature data are averaged over the months October-December. Results are shown only for stations where data are available for all events. As is evident in Fig. 5 (middle), the 3-month period following the peak in the stratospheric event is associated with temperature changes throughout Ant-

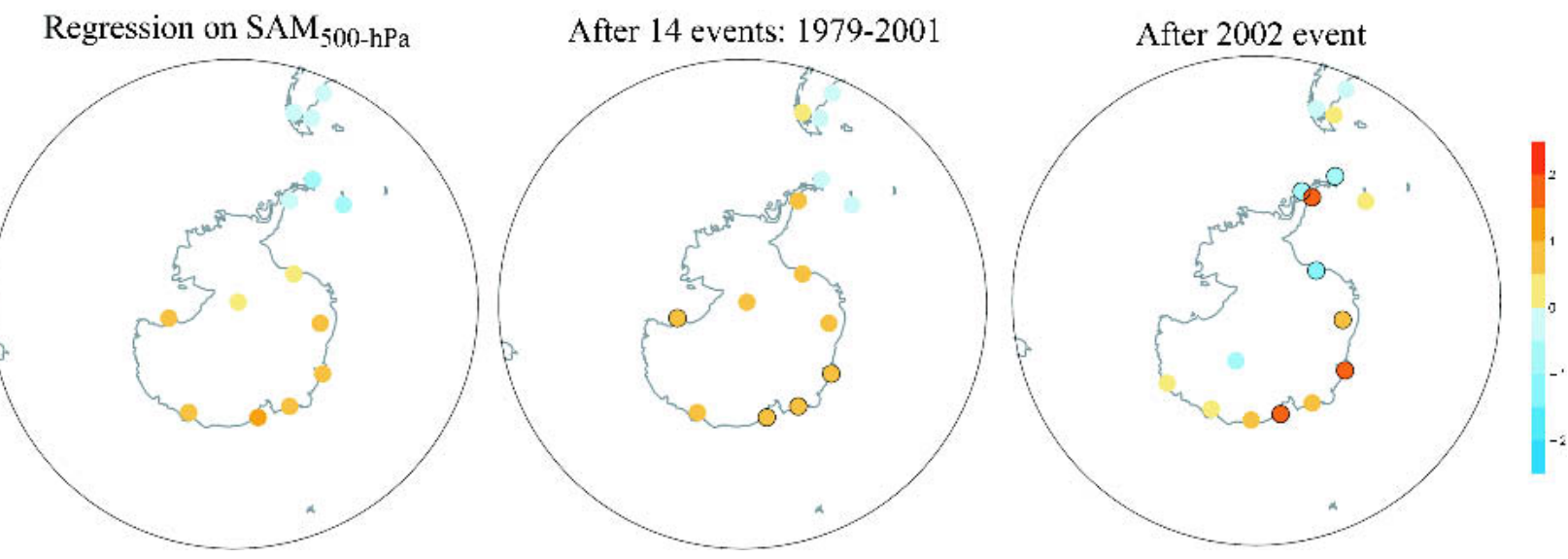

FIG. 5. As in Fig. 4, but for surface temperature anomalies. (middle and right) Composite differences during the 2-month period starting the first day of the first month following the onset of the weak and strong events listed in Table 1 . Units are ${ }^{\circ} \mathrm{C}$. Circles with black outlines denote regions where the results exceed (middle) the $95 \%$ confidence level and (right) 1 std dev about the climatological mean. 


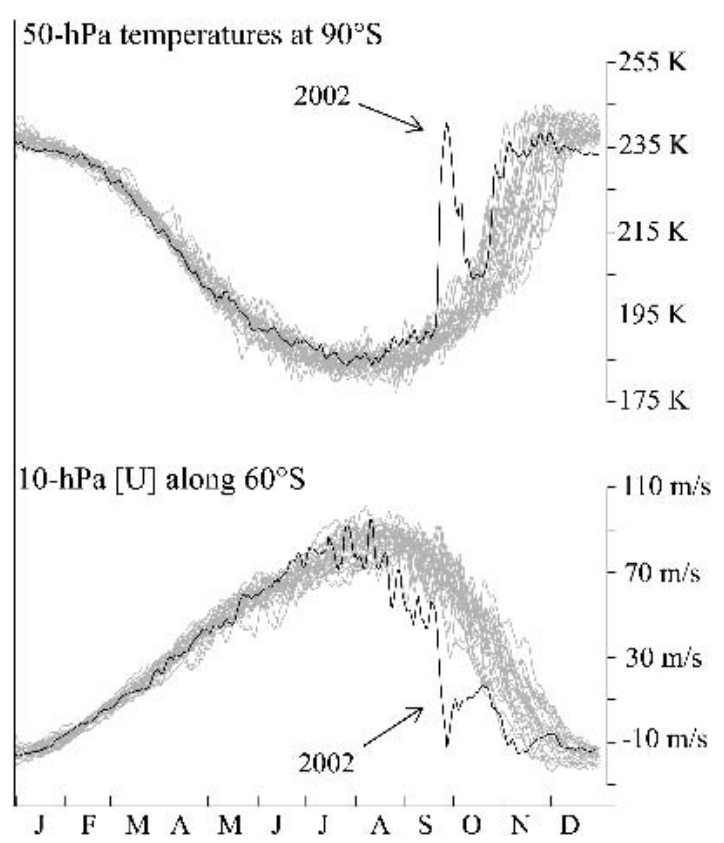

Fig. 6. (top) Temperature at $50 \mathrm{hPa}$ at the South Pole shown as a function of calendar day. Gray lines denote data for the years 1979-2001; the black line denotes data for the year 2002. (bottom) As in top, but for the zonal-mean zonal wind $([U])$ at $10 \mathrm{hPa}$ at $60^{\circ} \mathrm{S}$.

arctica that are broadly consistent with those found in association with the SAM. Over Antarctica, the changes in surface temperature are statistically significant at only 4 out of 11 individual stations (significance is indicated by the circles with black outlines). Nevertheless, the temperature changes are of the expected sign at 10 out of 11 Antarctic stations, and the area mean warming of $+0.75 \mathrm{~K}$ over eastern Antarctica and the Antarctic Plateau is statistically significant at the $99 \%$ confidence level.

\section{The SH SSW of 2002}

The results in the previous section demonstrate that large-amplitude anomalies in the SH stratospheric circulation are typically followed by similarly signed anomalies in the SH tropospheric circulation reminiscent of the SAM. In this section, we examine analogous results computed for the SH sudden stratospheric warming of 2002. The results in the previous section may be viewed as providing a basis for anticipating the state of the SH tropospheric circulation during the $\sim 3$ month period following the onset of this event.

As noted in papers throughout this issue, the $\mathrm{SH}$ SSW of 2002 was unusual in two respects: 1) the stratosphere was disturbed by substantial wave activity earlier in the year than is typical in the historical record (e.g., Newman and Nash 2005), and 2) the amplitude of the event is unique in the observational record. The extraordinary amplitude of the event is evidenced in Fig. 6, which shows scatterplots of the zonal-mean zonal flow at $10 \mathrm{hPa}$ along $60^{\circ} \mathrm{S}$ and at 50-hPa temperature at the Pole for 2002 (black line) superposed on data for the years 1979-2001 (gray lines). In both the zonalmean zonal flow at $10 \mathrm{hPa}$ along $60^{\circ} \mathrm{S}$ and at $50-\mathrm{hPa}$ polar temperatures, the event approached $\sim 10$ standard deviations about the climatological mean for the month of September.

Figure 7 shows daily values of the SAM index at all levels from 1 June 2002 to 31 December 2002. At 10 $\mathrm{hPa}$, the SAM index was at least 1 standard deviation below its long-term mean throughout most of July and by August 30, reached sustained values less than -2 standard deviations. In late September, the SAM index at $10 \mathrm{hPa}$ dropped from -2 to -9 standard deviations in a period of 7 days, reaching a peak value of -9.8 standard deviations at $10 \mathrm{hPa}$ on 27 September 2002. Anomalies lower than -2 standard deviations were evident in the stratosphere until December 2002.

The vertical profile of the SSW of 2002 bears consid-

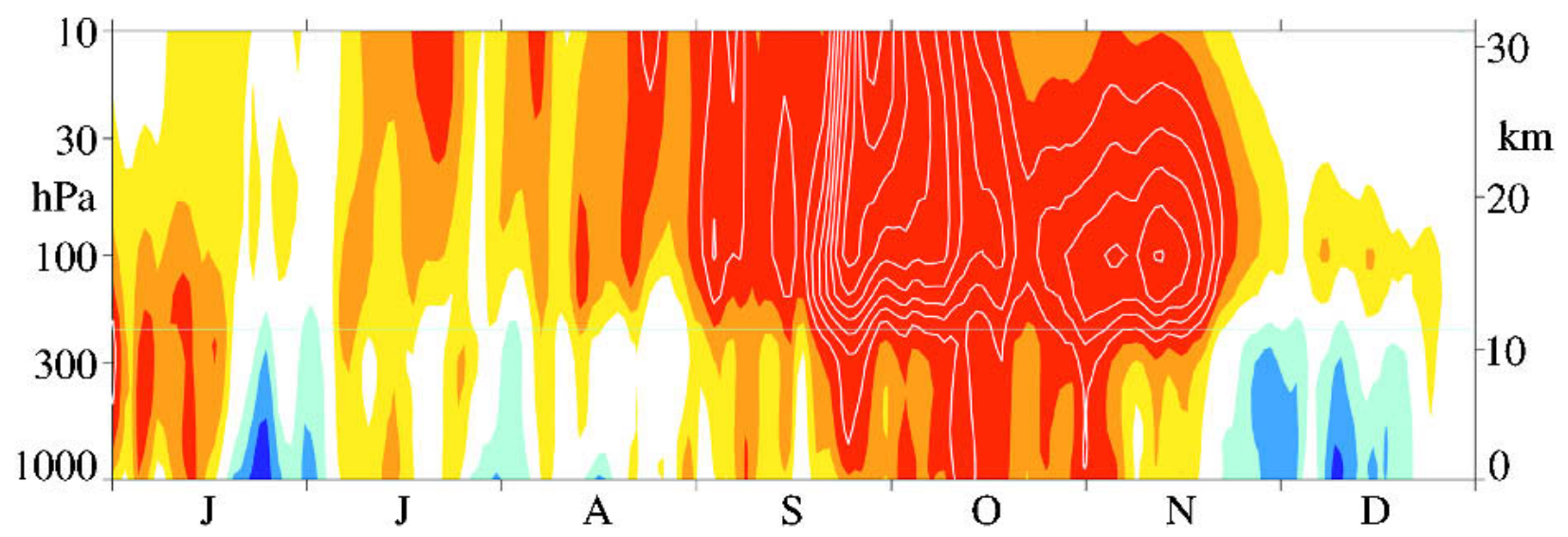

FIG. 7. Values of the SAM index during 2002. Contours are drawn at $0.5 \mathrm{std}$ dev. Shading is drawn for values exceeding $\pm 0.5 \mathrm{std}$ dev. Red shading denotes positive values in the SAM index (weaker-than-normal zonal flow along $60^{\circ} \mathrm{S}$ ). 
erable resemblance to the vertical profile for the 14 events considered in the previous section. In particular, the SSW of 2002 was associated with substantial anomalies that persisted from September to November not only at stratospheric levels but at tropospheric levels as well. The PDF of the SAM index at $500 \mathrm{hPa}$ was shifted 1.1 standard deviations below its climatological mean throughout the 10-90-day period following the 30 August onset of the SSW at $10 \mathrm{hPa}$ (Fig. 3, right). During this same period, the tropospheric circulation was marked by pronounced anomalies (in excess of one standard deviation) that strongly resemble the structure of the SAM (Fig. 4, right; the spatial correlation between the patterns in the right and left panels of Fig. 4 is $r=0.82$ ). Also consistent with the composites in the previous section, the months of September-November 2002 were associated with warmer-than-normal surface temperatures throughout much of eastern Antarctica and the Antarctic Plateau (Fig. 5, right). Indeed, at the South Pole itself, record high daily temperatures up to $-27^{\circ} \mathrm{C}$ occurred on 30 and 31 October 2002 (result based on daily data of 1957-2002 from the University of Wisconsin Space Science and Engineering Center). The enhanced amplitude of the anomalies in the tropospheric circulation following the SH SSW of 2002 is consistent with the unusual magnitude of this event.

\section{Concluding remarks}

The findings in this study add to an increasing body of observational evidence that suggests that the stratosphere plays an important role in driving tropospheric weather on the time scales longer than the limit of deterministic weather forecasts. In both the historical record (1979-2001) and during the SH SSW of 2002, largeamplitude anomalies in the SH stratospheric zonal flow are followed by substantial anomalies in the tropospheric circulation that persist as long as the overlying anomalies in the lowermost stratosphere for up to $\sim 3$ months. The shifts in the mean of the tropospheric circulation exhibit a high degree of statistical significance and are consistent with the observed "reddening" of the SH tropospheric circulation during the late spring season (Baldwin et al. 2003). The results thus lend credence to the hypothesis that photochemical ozone depletion has played a key role in driving recent climate change not only in the SH stratosphere, but in the SH troposphere as well (Thompson and Solomon 2002; Gillett and Thompson 2003).

The results presented in this study are remarkably similar to those observed in association with anomalies in the NH stratospheric polar vortex, as documented in Baldwin and Dunkerton (2001). Thus, the results provide independent verification for the observed relationships between long-lived anomalies in the NH stratosphere and the surface climate. One notable difference between the two hemispheres is the enhanced persis- tence of the $\mathrm{SH}$ anomalies. In the $\mathrm{NH}$, major stratospheric events are followed by anomalies in the lower stratosphere and troposphere that persist for up to $\sim 60$ days; in the $\mathrm{SH}$; such events are followed by anomalies in the lowermost stratosphere and troposphere that persist for up to $\sim 90$ days. The enhanced persistence of anomalies in the SH stratosphere is consistent with the relative dearth of dynamical forcing there.

Acknowledgments. We thank two anonymous reviewers for their helpful comments on the manuscript. DWJT is supported by the National Science Foundation under grants CAREER: ATM-0132190 and ATM0320959. MPB is supported by NSF's Climate Dynamics Program, NOAA's Office of Global Programs, and NASA's SR\&T Program for Geospace Sciences, Living With a Star Program, and Oceans, Ice, and Climate Program. SS appreciates the support of NOAA's Climate and Global Change Program.

\section{REFERENCES}

Baldwin, M. P., and T. J. Dunkerton, 1999: Propagation of the Arctic Oscillation from the stratosphere to the troposphere. J. Geophys. Res., 104, 30 937-30946.

— weather regimes. Science, 294, 581-584.

—, D. B. Stephenson, D. W. J. Thompson, T. J. Dunkerton, A. J. Charlton, and A. O'Neill, 2003: Stratospheric memory and extended-range weather forecasts. Science, 301, 636-640.

Charlton, A. J., and Coauthors, 2005: The splitting of the stratospheric polar vortex in the Southern Hemisphere, September 2002: Dynamical evolution and impacts on ozone. J. Atmos. Sci., 62, 590-602.

Charney, J. G., and P. G. Drazin, 1961: Propagation of planetaryscale disturbances from the lower into the upper atmosphere. J. Geophys. Res., 66, 83-109.

Gillett, N. P., and D. W. J. Thompson, 2003: Simulation of recent Southern Hemisphere climate change. Science, 302, 273-275.

Gong, D., and S. Wang, 1999: Definition of Antarctic Oscillation index. Geophys. Res. Lett., 26, 459-462.

Graversen, R. G., and B. Christiansen, 2003: Downward propagation from the stratosphere to the troposphere: A comparison of the two hemispheres. J. Geophys. Res., 108, 4780, doi:10.1029/2003JD004077.

Hartmann, D. L., 1976: The structure of the stratosphere in the Southern Hemisphere during late winter 1973 as observed by satellite. J. Atmos. Sci., 33, 1141-1154.

_ , and F. Lo, 1998: Wave-driven zonal flow vacillation in the Southern Hemisphere. J. Atmos. Sci., 55, 1303-1315.

Jones, P. D., 1994: Hemispheric surface air temperature variations: A reanalysis and update to 1993. J. Climate, 7, 17941802.

Kalnay, E., and Coauthors, 1996: The NCEP/NCAR 40-Year Reanalysis Project. Bull. Amer. Meteor. Soc., 77, 437-471.

Karoly, D. J., 1990: The role of transient eddies in low-frequency zonal variations of the Southern Hemisphere circulation. Tellus, 42A, 41-50.

Kidson, J. W., 1988: Indices of the Southern Hemisphere zonal wind. J. Climate, 1, 183-194.

Kuroda, Y., and K. Kodera, 1998: Interannual variability in the troposphere and stratosphere of the Southern Hemisphere winter. J. Geophys. Res., 103, 13 787-13 799.

Limpasuvan, V., and D. L. Hartmann, 2000: Wave-maintained annular modes of climate variability. J. Climate, 13, 4414 4429. 
- D. W. J. Thompson, and D. L. Hartmann, 2004: The life cycle of Northern Hemisphere sudden stratospheric warming. J. Climate, 17, 2584-2596.

Lorenz, D. J., and D. L. Hartmann, 2001: Eddy-zonal flow feedback in the Southern Hemisphere. J. Atmos. Sci., 58, 33123327.

McIntyre, M. E., 1982: How well do we understand the dynamics of sudden stratospheric warmings? J. Meteor. Soc. Japan, 60, 37-64.

Mechoso, C. R., 1988: Study of the stratospheric final warming of 1982 in the Southern Hemisphere. Quart. J. Roy. Meteor. Soc., 114, 1365-1384.

Newman, P. A., and E. R. Nash, 2005: The unusual Southern Hemisphere stratosphere winter of 2002. J. Atmos. Sci., 62, 614-628.

Orsolini, Y. J., C. E. Randall, G. L. Manney, and D. R. Allen, 2005: An observational study of the final breakdown of the
Southern Hemisphere stratospheric vortex in 2002. J. Atmos. Sci., 62, 735-747.

Shine, K. P., 1987: Middle atmosphere in the absence of dynamical heat fluxes. Quart. J. Roy. Meteor. Soc., 113, 603-633.

Shiotani, M., 1990: Low-frequency variations of the zonal mean state of the Southern Hemisphere troposphere. J. Meteor. Soc. Japan, 68, 461-471.

Thompson, D. W. J., and J. M. Wallace, 2000: Annular modes in the extratropical circulation. Part I: Month-to-month variability. J. Climate, 13, 1000-1016.

- and S. Solomon, 2002: Interpretation of recent Southern Hemisphere climate change. Science, 296, 895-899.

von Storch, H., and F. W. Zwiers, 1999: Statistical Analysis in Climate Research. Cambridge University Press, $494 \mathrm{pp}$.

Zhou, S. T., A. J. Miller, and J. K. Angell, 2002: Downwardpropagating temperature anomalies in the preconditioned polar stratosphere. J. Climate, 15, 781-792. 